\title{
Analytisch unbrauchbar? Eine Replik auf Andreas Brauns Kritik am Konzept der ,strukturellen Gewalt““
}

\author{
Peter Imbusch • Lotta Mayer
}

Eingegangen: 17. Juni 2021 / Überarbeitet: 9. Juli 2021 / Angenommen: 22. Juli 2021 / Online publiziert: 12. August 2021

(C) Der/die Autor(en) 2021

Andreas Brauns Aufsatz (Braun 2021) dient dem Nachweis der analytischen Unschärfe von Johan Galtungs Begriff der strukturellen Gewalt (Galtung 1969) und der dadurch bedingten Unbrauchbarkeit desselben für eine Gewaltsoziologie der Gegenwart. Braun hält den Begriff für ,,analytisch überschätzt“" und knüpft dabei an einen Aufsatz von Peter Imbusch an, der den Begriff eher für ,,analytisch unterschätzt“ hält (Imbusch 2017). Die kritische Auseinandersetzung mit Galtung ist natürlich ein legitimes Anliegen; darüber hinaus ist es auch verdienstvoll, eine neue Debatte über eine angemessene Soziologie der Gewalt zu führen. Verdienstvoll ist ebenfalls, dass Braun sowohl selbst an sehr aktuelle Beiträge aus der an Galtung anknüpfenden kritischen Konfliktforschung anschließt (u.a. Brunner 2020) als auch dazu anregen will, das Potenzial der ,situationalistischen' Gewaltforschung für die Friedens- und Konfliktforschung auszuloten. Schließlich laufen die Diskussionen in den hier angesprochenen Forschungssträngen - Gewaltsoziologie in der Tradition der ,Innovateur*innen“ (vgl. wegweisend von Trotha 1997) und des ,Situationalismus“ (wegweisend Collins 2008) einerseits, interdisziplinäre kritische Konfliktforschung andererseits - in der Regel gänzlich unverbunden nebeneinander her.

Dennoch möchte man an vielen Stellen von Brauns Text ansetzen, nachhaken, sie kritisch hinterfragen oder auch ihnen widersprechen. Wir beschränken uns auf drei unseres Erachtens zentrale Aspekte: die Verortung des Texts in der Kontroverse um verschiedene Gewaltbegriffe (1), Brauns zentrales Argument der analytischen Unbrauchbarkeit des Begriffs der strukturellen Gewalt aufgrund der ihm inhärenten Vermischung von Macht und Gewalt (2) und die Frage, inwiefern denn die situationistische Gewaltanalyse einen Erkenntnisfortschritt in der Gewaltsoziologie mit sich bringt (3).

Peter Imbusch $(\bowtie)$

Bergische Universität Wuppertal, Wuppertal, Deutschland

E-Mail: pimbusch@uni-wuppertal.de 


\section{Der Text als Zeichen einer polarisierten Debatte}

Zunächst einmal kann der Text selbst als eine Art ,Symptom ' gelesen werden, nämlich als Produkt und Zeichen der schon seit Jahrzehnten intensiv und kontrovers geführten Debatte um Gewaltbegriffe. Dass es derer verschiedene gibt, ist nicht weiter überraschend - und eigentlich auch gar nicht problematisch. So hat Dietrich Schotte jüngst sehr erhellend auf sprachphilosophischer Basis argumentiert, dass "Gewalt“ einen essentiell umstrittenen Begriff darstelle, d.h. einen Begriff, der sowohl einen deskriptiven als auch einen normativen Gehalt aufweist und dabei partiell offen und unbestimmt bleibt (Schotte 2020). Vielleicht sollte angesichts der unhintergehbaren normativen Dimension des Begriffs auch die Verve, mit der die Diskussion geführt wird, nicht weiter erstaunen. Dennoch ist es beizeiten befremdlich, wie diese Debatte geführt wird.

Zum einen ist die langjährige und immer wieder aufflammende Debatte um die "strukturelle Gewalt“ bereits insofern bemerkenswert, als sich der Begriff in der Galtungschen Version aufgrund seiner Schwächen und Unschärfen längst überlebt hat. Eben wegen seiner analytischen Unklarheiten wird er in weiten Teilen der sozialwissenschaftlichen Debatten zu Gewalt kaum noch verwendet. ${ }^{1}$ Auch Galtung selbst hat im Übrigen nie die Bedeutung von direkter physischer Gewalt als der eigentlichen Gewalt geleugnet, sondern nur andere Aspekte - wie strukturelle oder kulturelle Gewalt - hinzugefügt, um bestimmter Phänomene habhaft zu werden, die er für gewaltsam gehalten hat. Die Rangfolge möglicher Gewaltphänomene steht hier also völlig außer Frage - was man angesichts der Schärfe der Auseinandersetzungen manchmal vergessen könnte. Davon unberührt bleibt zunächst die Frage nach der Sinnhaftigkeit des Begriffs der strukturellen oder kulturellen Gewalt.

Zum anderen fällt auf, dass die Debatte um ebendiese Sinnhaftigkeit als eine Art Grabenkampf oder Glaubenskrieg mit fast schon religiösen Untertönen geführt wird: In der sozialwissenschaftlichen Gewaltforschung scheint es manchmal weniger um den Ertrag bzw. möglichen Erkenntnisgewinn eines bestimmten Paradigmas zu gehen als um das klare Bekenntnis zu der einen oder anderen Richtung - enger oder weiter Gewaltbegriff, „Mainstreamer“ oder „Innovateur*in“ (vgl. Imbusch 2004), Galtung oder Popitz -, mit dem dann der jeweils andere Gewaltbegriff als unbrauchbar zurückgewiesen wird. Die Logik ist dann die eines prinzipiellen Entweder-Oder, nicht die einer differenzierten Erörterung der Vor- und Nachteile, die die jeweiligen Begriffe für die Analyse unterschiedlicher Phänomene oder Aspekte der Gewalt zu bieten haben könnten.

Ohne hier einem instrumentalistischen Theorieverständnis das Wort reden zu wollen, ist es doch überraschend, dass weder eine offene Debatte über mögliche Anwendungsbereiche der einzelnen Begriffe noch eine über den Nutzen und Ertrag in systematisch vergleichender Perspektive geführt wird und die Frage, wie sie

\footnotetext{
1 Im Übrigen macht das auch der inkriminierte Aufsatz von Imbusch nicht. Dessen Anliegen war vielmehr, das Potenzial dieses Begriffs für eine kritische Gesellschaftstheorie auszuloten, und nur insofern bezeichnete er ihn als unterschätzt. Hier zeigen sich die Risiken einer selektiven bzw. eindimensionalen Rezeption von Beiträgen, die deren Argumentationsgänge primär als Steinbruch für eigene Argumente nutzt, selbst wenn dies der Zielsetzung des Autors des fraglichen Beitrags gar nicht entspricht.
} 
sich eventuell fruchtbar miteinander verbinden ließen, gänzlich außen vor bleibt. Letzteres verwiese ja gerade darauf, dass es Phänomene geben kann, deren Analyse einer ebensolchen Verbindung bedarf, oder vielleicht durch eine solche Verbindung sogar neue Erkenntnisse gewonnen werden könnten. Auch Brauns Aufsatz scheint eher dem Zweck zu dienen, aus einem aktuellen Anlass heraus nochmals eine explizite Begründung für eine (mittlerweile nicht mehr ganz so neue) Richtung der Gewaltsoziologie liefern zu wollen, die in den letzten Jahren viel Aufmerksamkeit erfahren hat. Indem der Beitrag sich primär dem Nachweis widmet, dass der Begriff der strukturellen Gewalt per se durch seine Unschärfe für die Gewaltsoziologie unbrauchbar sei bzw. nichts beitrage, was die (situationalistische) Gewaltsoziologie nicht sowieso schon täte, weist er sich selbst als durchaus typischen Vertreter seiner Gattung aus.

\section{Macht vs. Gewalt?}

Brauns Kernthese, dass das Konzept der strukturellen Gewalt unscharf ist, insbesondere nicht systematisch zwischen Gewalt, Macht und Herrschaft unterscheidet, ist weder neu noch überraschend noch ernsthaft umstritten. Dass daraus analytische Schwächen resultieren, ist folglich fast schon trivial. Hier rennt Braun letztlich recht offene Türen ein. Entsprechend fragt sich der Leser stellenweise, weshalb ein so großer argumentativer Aufwand betrieben wird, um ebendies zu zeigen. Die entscheidende Frage wäre doch vielmehr, was ggf. durch Galtungs Idee für die gegenwärtige Gewaltsoziologie zu gewinnen wäre (oder eben nicht), unter welchen Voraussetzungen einschließlich begrifflicher Modifikationen dies der Fall sein könnte und ob bzw. wie diese Bedingungen hergestellt werden können. Brauns Argumentation trägt dabei nur bedingt zur Aufklärung bei. Dies gilt sowohl für seine Explikation des Bezugsproblems als auch für seine Kontrastierung von Macht und Gewalt.

Zum erstgenannten Aspekt wäre zu sagen, dass Braun seinem Begriffsvergleich einen wissenschaftstheoretischen Exkurs voranstellt: Vergleichen lasse sich nur, was ein gemeinsames Bezugsproblem aufweise. Dem ist zwar uneingeschränkt zuzustimmen; doch es beantwortet nicht die Frage, wie man denn ein gemeinsames Bezugsproblem identifiziert. In dieser Hinsicht überzeugt Brauns Argumentation nicht: Er betrachtet physische Gewalt als das gemeinsame Bezugsproblem, weil Galtungs Argumentation eben dort ansetzt. Letzteres ist, wie oben bereits skizziert, unbestreitbar richtig - wenn es aber darum geht, das Konzept der strukturellen Gewalt (also: physische und psychische Verletzung ohne unmittelbares Handanlegen) mit einem engen Gewaltverständnis (also: direkte, intentionale körperliche Verletzung) $\mathrm{zu}$ vergleichen, dann kann nicht Gewalt im engen, physischen Sinn das gemeinsame Bezugsproblem der beiden Konzepte sein. Vielmehr muss das Gemeinsame an einer abstrakteren Stelle verortet werden: nämlich bei dem ,kleinsten gemeinsamen Nenner", auf den sich beide Konzepte bringen lassen. Und dies ist einfach die Schädigung von Menschen durch Menschen.

Dann erst lassen sich die beiden Ansätze sinnvoll vergleichend zueinander in Beziehung setzen. Nur so kann systematisch gefragt werden, inwiefern sich ihre zen- 
tralen Definitionen unterscheiden, d.h. vor allem: auf welche Art von Schädigung sie schauen (,enge' Gewaltbegriffe: physische Verletzung; Galtung: physische und psychische Beeinträchtigung, und zwar auch im Sinne unterbliebener möglicher Entwicklung), welche Arten der Verursachung der Schädigung sie begrifflich ein- oder ausschließen (,enge' Gewaltbegriffe: intentionale Schädigung durch ein handelndes Subjekt; Galtung: auch unintendierte und indirekte Schädigung ohne identifizierbare Verursacher*innen) und welche unterschiedlichen Themen- und Fragestellungen einerseits und Erklärungsansätze andererseits sich daraus ergeben. Derart erst können ihre relativen Stärken und Schwächen in Bezug auf unterschiedliche Forschungsgegenstände offen untersucht und beurteilt werden. Unterstellt man dagegen, wie Braun es tut, als gemeinsames Bezugsproblem den zentralen Gegenstand nur einer Forschungsrichtung, ist das Ergebnis, dass ebendiese Richtung in Bezug auf die Analyse des besagten Gegenstands überlegen sei, letztlich schon präjudiziert.

Zur Differenz zwischen Macht und Gewalt ist schon so viel geschrieben worden, dass das Nachzeichnen dieser Debatte an dieser Stelle weder möglich noch erforderlich ist. Entscheidend an dieser Stelle ist vielmehr, dass Braun, obwohl der Begriff der Macht in seiner Argumentation zentral ist, die von ihm selbst herangezogenen Ansätze von Weber und Popitz nur sehr kursorisch rekonstruiert und dabei deren Differenziertheit und Systematik nur bedingt gerecht wird. Daraus ergeben sich mehrere, miteinander zusammenhängende Probleme in seiner Argumentation.

So unterscheidet Braun nicht hinreichend klar zwischen dem, was Popitz „Aktionsmacht" nennt, also Handlungsmacht in einer Situation der Co-Präsenz, und ,autoritativer Macht“ bzw. Herrschaft: ${ }^{2}$ Die vorgenommene schematische Kontrastierung von „Macht“ und „Gewalt“ ergibt nur dann Sinn, wenn „Macht“ hier auch oder insbesondere „Herrschaft“ meint, nicht „Aktionsmacht“. Die späteren Ausführungen - etwa, dass Macht (oder vielmehr: Herrschaft) bei beiden Autoren Struktur sei und also als solche nicht handelt - bestätigen dies. Nun fragt sich allerdings zum einen, wieso dies ein Problem sein sollte - schließlich kann Herrschaft auch als Struktur gewaltsoziologisch relevant sein, wenn man die Gewaltsoziologie nicht auf eine mikrologische Herangehensweise reduzieren will. Zum anderen hat Herrschaft bei Weber und Popitz ganz zentral etwas mit Legitimität zu tun. Das weiß Braun natürlich, lässt diesen Aspekt aber an der besagten Stelle dennoch völlig außen vor (und diskutiert ihn bei „kultureller Gewalt“ nur hinsichtlich sehr spezifischer Aspekte). Es wäre aber auch gewaltsoziologisch durchaus lohnend (falls nicht sogar erforderlich), sauber zwischen dem Verhältnis von nicht-gewaltsamen Formen bloßer (Handlungs-)Macht und physischer Gewalt auf der einen Seite und der Relation von legitimer Herrschaft bzw. legitimen Herrschaftsstrukturen und physischer Gewalt auf der anderen Seite zu unterscheiden (und darüber hinaus danach zu fragen, wie eigentlich physische Gewalt selbst legitimiert wird).

Brauns Popitz-Exegese erscheint auch noch in einer anderen Hinsicht als selektiv: Popitz fasst unter dem Konzept der „Aktionsmacht“ Handlungen, die den anderen schädigen, sei es in seiner sozialen Teilhabe, in materieller Hinsicht oder körperlich als physische oder psychische Gewalt (Popitz 1992, S. 44). Gewalt ist

\footnotetext{
${ }^{2}$ Die weiteren von Popitz unterschiedenen, aufeinander irreduziblen Machtformen - instrumentelle und datensetzende Macht - bleiben zudem gänzlich außen vor.
} 
damit ein ganz bestimmter Typus von Aktionsmacht, sie kann also als eine Form der Machtausübung verstanden werden. Vor diesem Hintergrund aber erscheint die grundsätzliche Kontrastierung von Macht und Gewalt, wie sie seit Hannah Arendt hoch im Kurs steht, fraglich (und die stillschweigende Verschiebung der Argumentation hin zur Herrschaft als ein Versuch, die Argumentation irgendwie zu retten). Schließlich bedeutet ,begriffliche Klarheit‘ nicht nur die Abgrenzung von Begriffen voneinander, sondern auch die Explikation ihrer Bezüge zueinander.

Zudem wird die Interpretation von struktureller Gewalt als Macht bzw. Herrschaft auch Galtungs Konzept selbst nicht gerecht: Herrschaft, die Braun als Kern des Konzepts identifiziert, ist ja keineswegs deckungsgleich mit „sozialer Ungleichheit“ in ihrer ganzen Breite und Varianz, auch wenn sie natürlich damit zu tun hat. Galtungs Konzept der strukturellen Gewalt zielt aber gerade sowohl auf soziale Ungleichheit als komplexem Phänomen als auch auf dessen Verbindungen mit Herrschaftsstrukturen - ohne die beiden Phänomene miteinander gleichzusetzen.

Brauns Argumentation weist damit sowohl hinsichtlich der Exegese von Weber und Popitz als auch der von Galtung Schwächen auf. Ob sich auf dieser theoretischen Basis das Argument, dass Macht und Gewalt strikt zu unterscheiden und aufgrund des vermeintlichen Mangels an diesbezüglicher Unterscheidung Galtungs Begriff der strukturellen Gewalt analytisch für die Gewaltsoziologie unbrauchbar sei, so einfach aufrechterhalten lässt, erscheint doch eher fraglich.

\section{Mögliche Erträge eines unscharfen Konzepts}

Braun zufolge ist aus Galtungs Konzept der strukturellen Gewalt für die Gewaltsoziologie nichts zu gewinnen. Zu der Frage, ob eventuell ein anders gefasster Begriff der strukturellen Gewalt - der von Galtung zwar die Inspiration bezieht, nicht aber das problematische Konzept in all seinen Details, Unschärfen und Inkonsistenzen reproduziert - Abhilfe schaffen könnte, stößt er gar nicht erst vor. Gegen Imbuschs Argument, das Konzept rege an zum Blick auf Ursachen und Kontextbedingungen von Gewalt - sprich: zum Blick über die „Gewaltsituation“ hinaus -, stellt er die These, dass verschiedene jüngere Ansätze der soziologischen Gewaltdebatte ebendiese Desiderata in den Blick nähmen.

Wirft man allerdings einen näheren Blick auf die von Braun genannten Ansätze, so wird doch deutlich, dass damit noch lange nicht ,alles gesagt ' ist: Erstens sind diese Ansätze allesamt noch relativ jungen Datums, keineswegs unumstritten und ihre weitere Rezeption ungewiss. Zweitens wird die ,neuere Gewaltsoziologie“ hier von Braun vielleicht auch als unitarischer dargestellt, als sie tatsächlich ist (Trutz von Trotha ist nicht Randall Collins ist nicht Gesa Lindemann ist nicht Jan Philipp Reemtsma - auch das weiß Braun natürlich sehr gut, stellt es hier jedoch hinten an). Mit dem gemeinsamen Fokus auf physische Gewalt geht schließlich nicht zwingend auch eine sonstige Übereinstimmung einher, und entsprechend können die in den unterschiedlichen Strömungen berücksichtigten „Kontextbedingungen“ nicht ohne weiteres miteinander integriert werden. Drittens nehmen viele der von Braun hier angeführten Ansätze - insbesondere diejenigen, die sich selbst tatsächlich im situationalistischen Paradigma bewegen (etwa Koepp und Schattka 2020) - nur aus- 
gewählte „Kontextbedingungen“ in den Blick, und auch dies nicht allzu tiefgehend, geschweige denn systematisch. ${ }^{3}$ Aus der Binnenperspektive der situationalistischen Gewaltsoziologie mag es also vielleicht so aussehen, als ob das Thema Kontextualisierung schon ,abgefrühstückt“ sei. Nimmt man dagegen eine Außenperspektive ein, die von einer breiteren Sozialtheorie oder umfassenderen Phänomenzusammenhängen ausgeht und derart etwa soziale Ungleichheit oder den sozialen Konflikt in den Mittelpunkt rückt, dann erscheint der Gehalt der jüngeren gewaltsoziologischen Debatte eher als begrenzt und isoliert: Er bezieht nur ganz sporadisch Aspekte ein, die in der allgemeineren Soziologie und/oder in thematisch verwandten (interdisziplinären) Forschungsfeldern wie der Friedens- und Konfliktforschung als relevant für die Erklärung sozialen Handelns im Allgemeinen oder aber spezifischer gerade für die Entstehung von Gewalt (etwa in sozialen Konflikten) gelten.

Notwendig und u.E. zu allseitigem Nutzen wäre daher eine systematische Verknüpfung der unterschiedlichen Forschungsfelder. Ein vielversprechender Anfang dafür wäre, nach den vielfältigen, wechselseitigen und komplexen Beziehungen zwischen Macht, Herrschaft, sozialer Ungleichheit und Gewalt zu fragen. All diese Verhältnisse in ihrer Wechselseitigkeit sauber auszubuchstabieren und dabei dennoch Gewalt als Handlung nicht aus dem Auge zu verlieren, wäre durchaus eine lohnende Aufgabe für die soziologische Gewaltforschung. Genau darauf aber verweist letztlich Galtungs Konzept der strukturellen Gewalt.

Ob nun Galtungs - teils inkonsistentes, oft scherenschnittartiges, letztlich unklares und normativ aufgeladenes - Konzept der strukturellen Gewalt der beste Ansatz ist, um diesen komplexen Beziehungen nachzuspüren, darf dabei füglich bezweifelt werden. Insofern ist Brauns Skepsis durchaus berechtigt. Doch die in Galtungs Konzept enthaltene Anregung, Mahnung oder vielleicht auch Zumutung, eine sozialwissenschaftliche Gewaltanalyse eben nicht nur auf das Moment des unmittelbaren physischen Gewalthandelns zu beschränken, einfach dadurch zurückzuweisen, dass man auf begriffliche Probleme des Konzepts einerseits und verstreute gewaltsoziologische Arbeiten andererseits verweist, das ist dann doch zu kurz gesprungen.

Funding Open Access funding enabled and organized by Projekt DEAL.

Open Access Dieser Artikel wird unter der Creative Commons Namensnennung 4.0 International Lizenz veröffentlicht, welche die Nutzung, Vervielfältigung, Bearbeitung, Verbreitung und Wiedergabe in jeglichem Medium und Format erlaubt, sofern Sie den/die ursprünglichen Autor(en) und die Quelle ordnungsgemäß nennen, einen Link zur Creative Commons Lizenz beifügen und angeben, ob Änderungen vorgenommen wurden.

\footnotetext{
3 Letzteres würde auch voraussetzen, auf der Basis einer Sozialtheorie zu argumentieren, die eben nicht die mikrosoziologische Collinssche Sozialtheorie (vgl. Collins 2004) ist, welche der dominanten, situationalistischen Strömung in der gegenwärtigen Gewaltsoziologie zugrunde liegt. Zugespitzt formuliert ließe sich fragen, ob es vielleicht eher sozialtheoretische Engführungen sind, die zur gegenwärtigen Fokussierung des dominierenden Teils der Gewaltsoziologie auf die Situation des Gewalthandelns in Co-Präsenz führen, als tatsächlich gewaltsoziologische Gründe. Und ebenso ließe sich fragen, in welchen Hinsichten genau eigentlich die sehr enge Fokussierung auf „die Situation“ in der derzeitigen gewaltsoziologischen Debatte wirklich trägt und das Potenzial hat, die Gewaltforschung und auch die interdisziplinäre Konfliktforschung entscheidend voranzubringen - eine Diskussion, die derzeit beginnt (vgl. insbes. die Beiträge in Mittelweg 36, Band 1-2/2019).
} 
Die in diesem Artikel enthaltenen Bilder und sonstiges Drittmaterial unterliegen ebenfalls der genannten Creative Commons Lizenz, sofern sich aus der Abbildungslegende nichts anderes ergibt. Sofern das betreffende Material nicht unter der genannten Creative Commons Lizenz steht und die betreffende Handlung nicht nach gesetzlichen Vorschriften erlaubt ist, ist für die oben aufgeführten Weiterverwendungen des Materials die Einwilligung des jeweiligen Rechteinhabers einzuholen.

Weitere Details zur Lizenz entnehmen Sie bitte der Lizenzinformation auf http://creativecommons.org/ licenses/by/4.0/deed.de.

\section{Literatur}

Braun, Andreas. 2021. Strukturelle Gewalt - ein analytisch überschätzter Begriff. ZeFKo https://doi.org/ 10.1007/s42597-021-00057-1.

Brunner, Claudia. 2020. Epistemische Gewalt. Wissen und Herrschaft in der kolonialen Moderne. Bielefeld: transcript.

Collins, Randall. 2004. Interaction Ritual Chains. Princeton u. a.: Princeton University Press.

Collins, Randall. 2008. Violence. A Micro-Sociological Theory. Princeton u. a.: Princeton University Press. Galtung, Johan. 1969. Violence, Peace, and Peace Research. Journal of Peace Research 6:167-191.

Imbusch, Peter. 2004. „Mainstreamer“ versus „Innovateure“ der Gewaltforschung. Eine kuriose Debatte. In Gewalt. Entwicklungen, Strukturen, Analyseprobleme, Hrsg. Wilhelm Heitmeyer, Hans-Georg Soeffner, 125-148. Frankfurt a. M.: Suhrkamp.

Imbusch, Peter. 2017. „Strukturelle Gewalt“. Plädoyer für einen unterschätzten Begriff. Mittelweg 36(26):28-51.

Koepp, Tabea, und Chris Schattka. 2020. Eine kuriose Debatte wiederholt sich. Plädoyer für eine kontextsensible Mikrosoziologie der Gewalt. Österreichische Zeitschrift für Soziologie 45:23-42. https://doi. org/10.1007/s11614-020-00407-y.

Popitz, Heinrich. 1992. Phänomene der Macht. Tübingen: Mohr Siebeck.

Schotte, Dietrich. 2020. Was ist Gewalt? Philosophische Untersuchung zu einem umstrittenen Begriff. Frankfurt/Main: Klostermann.

Trotha, Trutz von. 1997. Zur Soziologie der Gewalt. In Soziologie der Gewalt Kölner Zeitschrift für Soziologie und Sozialpsychologie (Sonderheft Nr. 37)., Hrsg. Trutz von Trotha, 9-56. Opladen: Westdeutscher Verlag. 